\title{
Representation activity of the right and left hemispheres of the brain
}

\author{
N.N. Nikolaenko, A.Y. Egorov and E.A. Freiman \\ I.M. Sechenov Institute of Evolutionary Physiology and Biochemistry, Russian \\ Academy of Sciences, 44 Moris Thorez Prospect, 194223 St Petersburg, Russia
}

Correspondence to: N.N. Nikolaenko at above address

\begin{abstract}
Drawings by psychiatric patients were studied in various states (i) in depression; (ii) after neuroleptic injection; and (iii) during left hemisphere suppression induced by unilateral electroconvulsive seizure (UES). In these states, right hemisphere activation predominates. The results of the study demonstrate that, under the predominance of right hemisphere activation over the left hemisphere, there is a tendency to reproduce the image of the object and to represent it in near space. Drawings by psychiatric patients were also investigated in (i) the manic state; (ii) after injection of psychotropic drugs which improved the mood; and (iii) during right hemisphere suppression following right-sided UES. Under these conditions, left hemisphere activation predominates and the drawings loose the illusion of three-dimensional space. A tendency to reproduce the knowledge and the ideas of the object and to represent it in distant space was observed. Thus, both hemispheres may represent space and elaborate perceptive and conceptional models of the world in different ways. It is probable that different types of representation are based on global (right-hemispheric) in comparison with focal (left-hemispheric) attention to space.
\end{abstract}

Keywords: Cerebral asymmetry - Depression - Mania - Psychotropic drugs - Unilateral electroconvulsive seizures

\section{INTRODUCTION}

Drawings are one of the methods of neuropsychological examination of patients with organic lesions of the right and left hemispheres. However, traditional drawing analysis, such as estimation of drawing disability and presence of constructive apraxia, has limited applications. The hypothesis suggesting the predominant role of the right hemisphere in incorporating spatial information into drawing performance, leading to disproportion and faulty articulation of parts of the drawing, has been proposed. The left hemisphere was found to be dominant in the executive component of drawing, i.e. in planning the drawing process, leading to simplified versions of the model (Piercy et al., 1960; Arrigoni and De Renzi, 1964; Warrington et al., 1966).

From the point of view of this study, this hypothesis may need to be revised. A method is required to check the drawing abilities of the left hemisphere in conditions when a patient could draw only with the right hand before damage to the left hemisphere occurred. It is obvious that new approaches to investigate drawing abilities of the hemispheres should be developed. In this connection, models of transient suppression of one hemisphere are of interest. It is suggested that both hemispheres may represent space and elaborate perceptive and conceptional models of the world in different ways.

In the 1980s, an hypothesis was proposed in our laboratory. This hypothesis suggested that each hemisphere employed its own sign model of the world (Balonov et al., 1984; Nikolaenko and Deglin, 1974). The mechanisms of direct isomorphic perception of reality which are based on the iconic sign system, i.e. on direct images, are concentrated in the right hemisphere. The left hemisphere constructs a symbolic sign system which secures conceptual reflection of reality. Constant interaction and interference of the sign systems appears to be one of the major principles of mental activity.

This study attempts to elucidate how visual-sensual spatial relations (i.e. the visible geometry of objects) and knowledge of the spatial features of an object (i.e. objective geometry) are reflected in the drawings of (i) patients under conditions of right or left hemisphere suppression produced by UES; (ii) psychiatric patients after psychotropic drug administration; and (iii) patients in manic or depressive states. 


\section{METHODS}

An approach elaborated by Rauschenbach (1980) was employed to analyse drawings. Rauschenbach showed that there are two methods of three-dimensional space representation in a plane. The first method involves the representation of the visible geometry of objects; these are drawings in which an illusion of three-dimensional space (volume and depth) is created This method is employed in art to reproduce the visible form of objects. If the geometry of objects changes, depending on foreshortening, representation of visible geometry reflects perceptive space. A method of space projection which differs in principal is the representation of the geometry of the object in objective space in which the illusion of three-dimensional space is lost. This method of projection is, to a large extent, embodied in the practice of technical drawing for reproducing the form of an object without any distortions. It is known that representation of an object in a single plane is a property of every technical drawing; the planar approach is based on the method of orthogonal projections. Thus, this approach allows us to understand which space (objective or perceptive) is being represented by the subject. What is predominant for him in the representation activity, 'to know' or 'to see'?

Three experimental series were conducted. In all the experimental series, the subject was asked to draw 'by imagination' a person, a flower, a house, a table, a cube, a bridge over a river, two houses (one close to the subject and the other in the distance) and rails receding into the distance.

The protocol of the study was approved by the Research Council of I.M. Sechenov Institute of Evolutionary Physiology and Biochemistry and by the Ministry of Health of the Russian Federation.

\section{Effects of UES on drawings}

In this series, 62 depressed patients, diagnosed with bipolar affective disorder, recurrent depressive disorder and schizoaffective disorder, depressive type, according to ICD-10, and 30 patients diagnosed with paranoid schizophrenia according to ICD-10 were investigated. In total, there were 70 female and 22 male patients, aged between 21 and 45 years who were treated by UES. The patients were investigated 15 to 20 minutes after cessation of UES. To produce UES, electrodes were placed on the temporal area of the head, either on the left or on the right. It has been shown that such seizures produce a transient (30 to 60 minute) suppression of activity of the stimulated hemisphere, whereas functions of the non-stimulated hemisphere were restored rapidly and can be enhanced in a reciprocal way (Balanov et al., 1979; Deglin et al.,
1987). In each patient, right- and left-sided UES were administered on alternate days, allowing the comparison of the effects of suppression of the right and left hemispheres in the same subject as well as the comparison with data obtained before treatment (control).

\section{Effects of psychotropic drugs on drawings}

In this series, 90 patients ( 63 female and 27 male, aged between 18 and 50) were investigated following the administration of psychotropic drugs. Firstly, patients suffering from various forms of depression, diagnosed with bipolar affective disorder, recurrent depressive disorder or schizoaffective disorder, according to ICD10 , were given intravenous injections of drugs which improved mood. These were diazepam, 20 to $30 \mathrm{mg}$; imipramine, 50 to $75 \mathrm{mg}$; and sodium amytal, 250 to $300 \mathrm{mg}$. Secondly, manic patients, diagnosed with bipolar affective disorder or schizoaffective disorder, manic type, and schizophrenics, diagnosed with paranoid schizophrenia, were given injections of neuroleptics at the following doses: chlorpromazine, 50 to $100 \mathrm{mg}$; and haloperidol, 2.5 to $5 \mathrm{mg}$. It should be noted that neuroleptics can produce depression as a side effect. Investigations were carried out before injection with the drug (control) and 30 to 40 minutes after the injection. Using original psychosensory techniques (measurement of poststimulatory auditory adaptation in the left and right ears and estimations of visual images, which permitted the estimation of activation of each hemisphere separately, together with especially elaborated neuropsychological grid tests, it has been shown that injection of drugs which improved the mood was followed by a shift of activation balance towards the left hemisphere (Egorov, 1989; Egorov and Nikolaenko, 1992). Meanwhile, injection of neuroleptics resulted in the prevalence of right hemisphere activation over the left one (Egorov, 1989; Egorov and Nikolaenko, 1992).

\section{Effects of affective disorders on drawings}

In this series, 35 depressed patients (diagnosed with bipolar affective disorder, depressive episode, recurrent depressive disorder or schizoaffective disorder, according to ICD-10) and 22 manic patients (diagnosed with bipolar affective disorder or schizoaffective disorder), were investigated. This series included 50 female and 7 male patients, aged between 19 and 62 . The patients were drug free for at least 48 hours before the experiment.

Preponderance of the right over the left hemisphere in depression has been demonstrated by EEG analysis (Flor-Henry et al., 1979; Flor-Henry, 1983; Davidson, 1991), investigations of lateral eye movements 
(Schweitzer, 1979), dichotic listening (Wexler and Heninger, 1979) and psychosensory methods (Asadova, 1985; Egorov, 1986). Left hemisphere preponderance in mania has been demonstrated in our laboratory using psychosensory methods (Asadova, 1985; Egorov, 1986). This view is supported by Flor-Henry (1983).

Independent experts made blind estimations of which kind of representation, either visible or objective geometry, was present in the drawings. They had to estimate the presence of characteristic features of each type of representation in each drawing. The results were analysed statistically using the paired Student's t-test.

\section{RESULTS}

\section{Effects of UES on drawings}

Left hemisphere suppression. In this condition, patients preferred to represent the volume of objects. This includes those patients who, under control conditions, reproduced these objects in a single plane. Usually, the patients indicated volume by overlapping the distant parts of an object with the near ones. The patients represented objects as they have been envisioned routinely. These drawings were characterised by unbroken lines, clear outline, combining of parts into a whole and precise spatial relationships between the parts of the represented object. Therefore, compactness of the

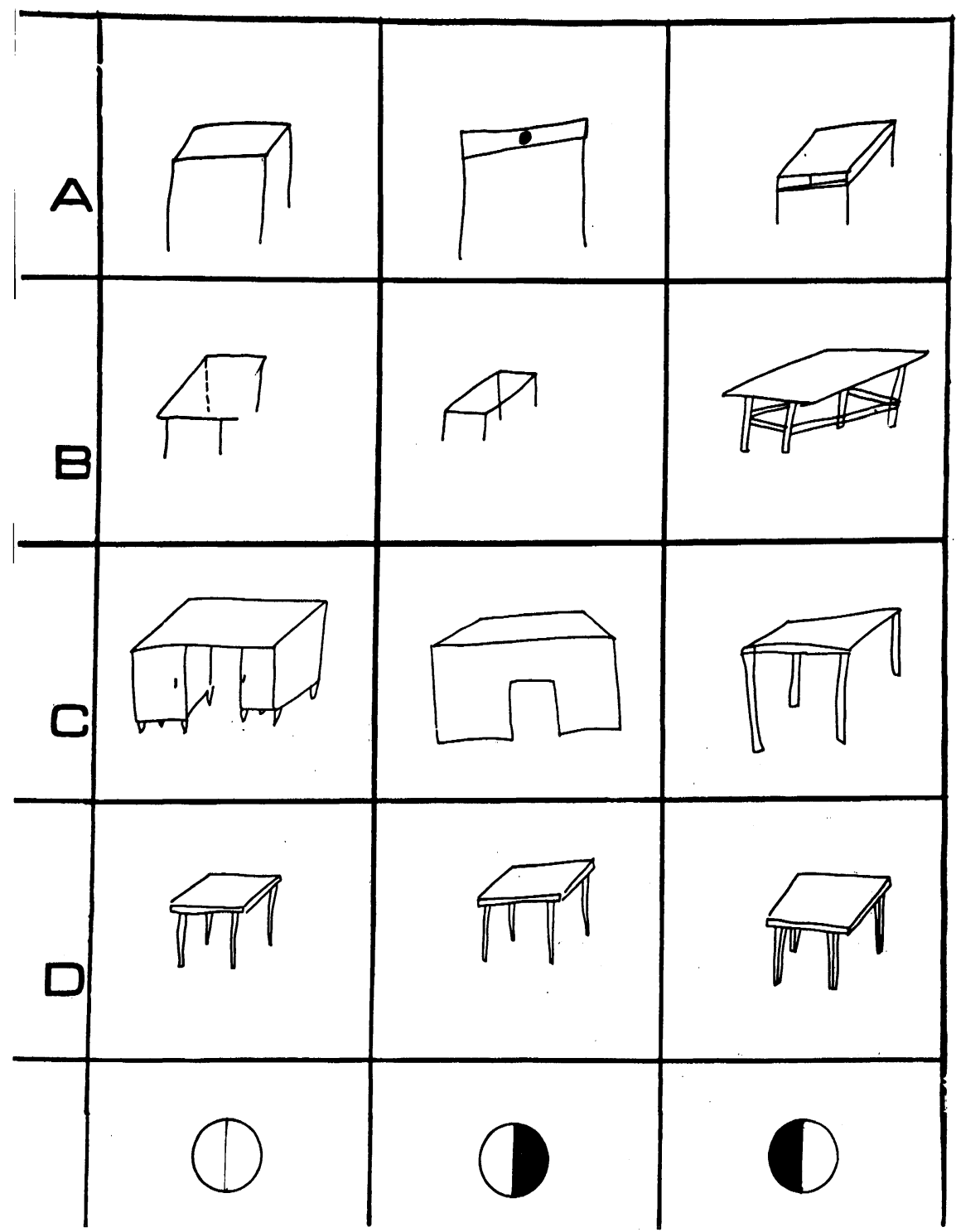

FIG. 1. Drawings of a table by four patients (A, B, C, D) under control conditions and after right and left UES The suppressed hemisphere is shaded. 
TABLE I. Frequency (\%) of representations of visible and objective geometry of space after UES

\begin{tabular}{lccc}
\hline Type of representation & Control & $\begin{array}{c}\text { After left } \\
\text { UES }\end{array}$ & $\begin{array}{c}\text { After right } \\
\text { UES }\end{array}$ \\
\hline Representation of visible geometry & $51 \pm 4$ & $72 \pm 4^{\star \star \star}$ & $29 \pm 4^{\star \star \star}$ \\
Representation of objective geometry: & $37 \pm 4$ & $24 \pm 4^{*}$ & $51 \pm 4^{*}$ \\
Orthogonal projections & $4 \pm 2$ & $1 \pm 1$ & $12 \pm 2^{*}$ \\
Reamers & $8 \pm 2$ & $3 \pm 1^{*}$ & $8 \pm 2$ \\
Representation of invisible outline & & \pm 2 \\
\hline
\end{tabular}

${ }^{\star * *} p<0.001,{ }^{* *} p<0.01,{ }^{\star} p<0.05$ in comparison with controls.

form was emphasised, an impression of image integrity was produced and the material nature of the objects was represented. The synthesis of different projections by combination of the side view and view from above in an integrated image and continual transition from one projection to another was also typical. This resulted in the ability to integrate a large number of signs of the object, as they were seen from different viewpoints, in the process of 'mentally rotating' the object.
While reproducing objects in space at close quarters, such as in drawings of a table and a cube, the representations were increased in size, as if the objects were reproduced from a close viewpoint. Objects were represented as if they were seen at a short distance, without pronounced perspective reduction. Moreover, in this situation, a reversed perspective was apparent in some drawings (62 $\pm 5 \%$ in comparison with $22 \pm 5 \%$ under control conditions, $p<0.001$ ) (Figure 1). With such a method of three-dimensional space projection

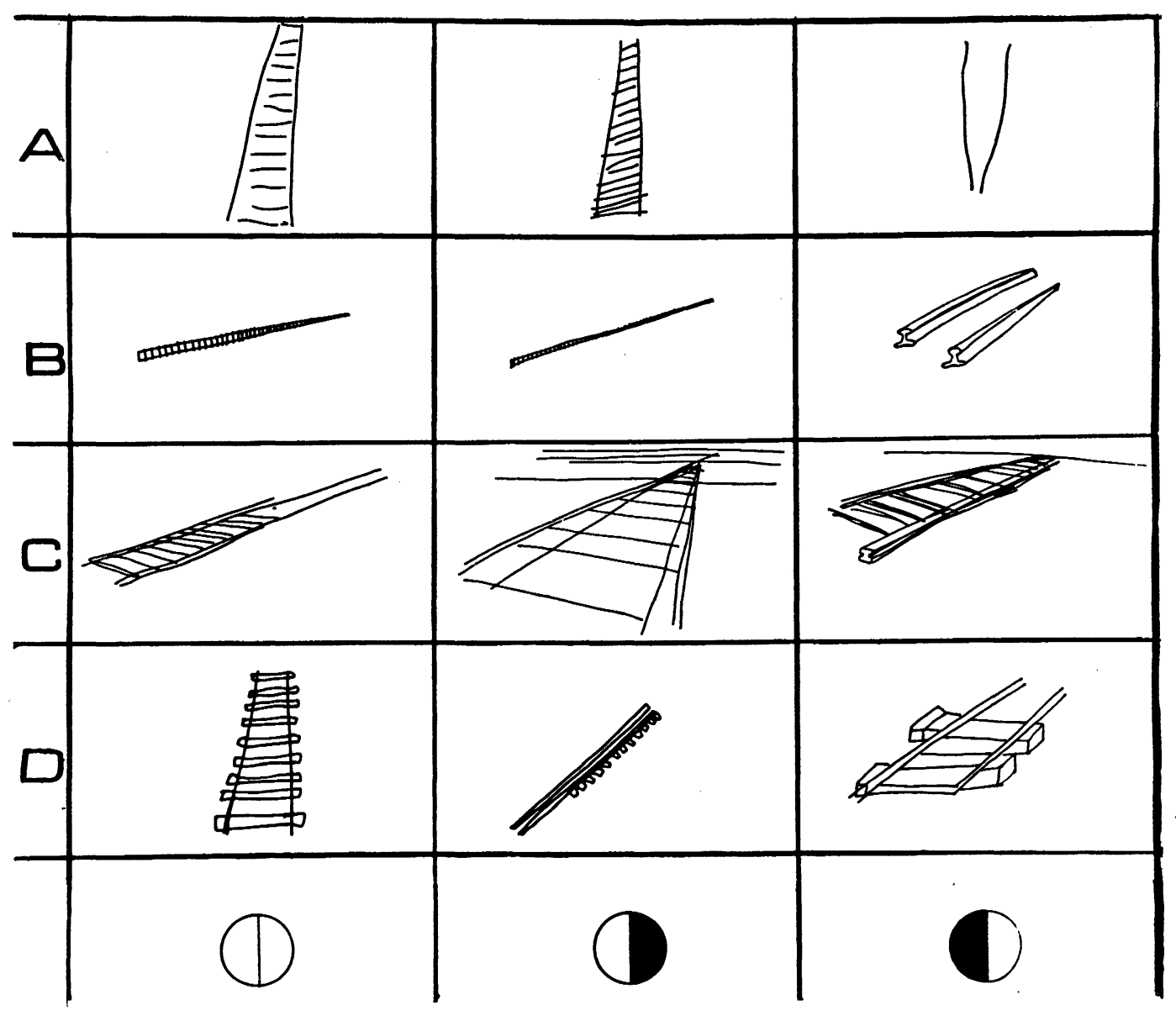

FIG. 2. Drawings of rails receding into the distance by four patients $(A, B, C, D)$ under control conditions and after left and right UES. The suppressed hemisphere is shaded. 

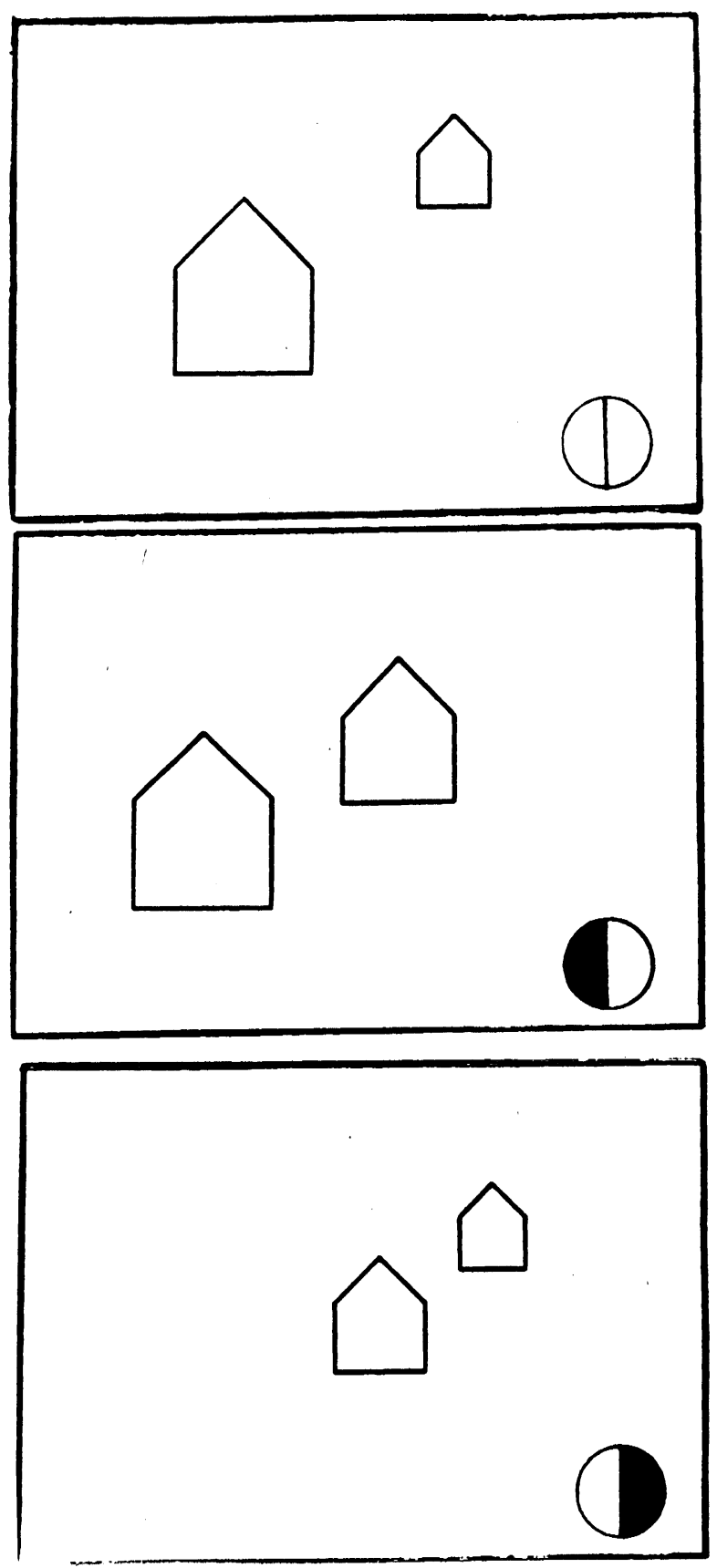

FIG. 3. Drawings of two houses, one at close quarters and the other in the distance. The drawings are reproduced using measurements of the drawing area as well as the position of the drawings on the paper. These drawings represent averages for 22 control patients, 27 patients with left hemisphere suppression and 31 patients with right hemisphere suppression. The suppressed hemisphere is shaded.

the visible form of an object was represented convincingly. The vast majority of drawings were estimated as representations of visible geometry according to Rauschenbach (1980); Table I.
While reproducing an integrated extended space, such as rails receding into the distance, the patients had an increased tendency to emphasise the volume of objects in the near part of the space. At the same time, the distant part of the space was limited (Figure 2). In the drawings of a house at close quarters and a house in the distance, both images were shifted downwards and to the left on the sheet of paper. There was a marked shift in the image of the house in the distance. The distance between the two houses decreased (Figure 3). In comparison with control data, the representation of a house in the distance increased in size significantly $(p<0.01)$. When the differences in size and distance are ignored, there is a shift of both images to the near part of the space.

Right hemisphere suppression. In this condition, representations were distinguished by their planar character and volume signs disappeared, even in those patients who had represented objects as volumetric under control conditions. Usually, a set of drawing methods was employed to make the representations more informative (Table I). These were: (i) orthogonal projections which appeared even in representations of a cube and a table; they were represented as rectangles or rhombi, Figure 4; (ii) reamer, i.e. the combination of orthogonal projections of different sides of the object on the same drawing; (iii) representation of the invisible outline of the object which allowed the patient to add some information about the object; (iv) section, such as representation of the sectional river-bed (Figure 5), which reflected knowledge of the construction of the object; and (v) conventional shift of parts of the represented object which allowed the objective geometry of the object to be reproduced, such as the number and equal size of table legs. Thus, retained activity of the left hemisphere is characterised by objective representation of geometry in which the illusion of volume is lost.

In the condition of right hemisphere suppression, a baseline was often incorporated into a drawing of a tree or a flower. The baseline is a horizontal line below which the roots of the plant were represented. Such drawings were shifted upwards and the space below the baseline became free. As the result, the space on the sheet of paper was sectioned: top (the plant) - bottom (underground space). Of interest is that the baseline, which is a conventional combination of the surface of the earth and the horizon, serves as a reliable sign of representation of the objective geometry of the space with the help of orthogonal projections (Rauschenbach, 1980).

Space section in the horizontal direction was also noted. Under right hemisphere suppression, geometri- 


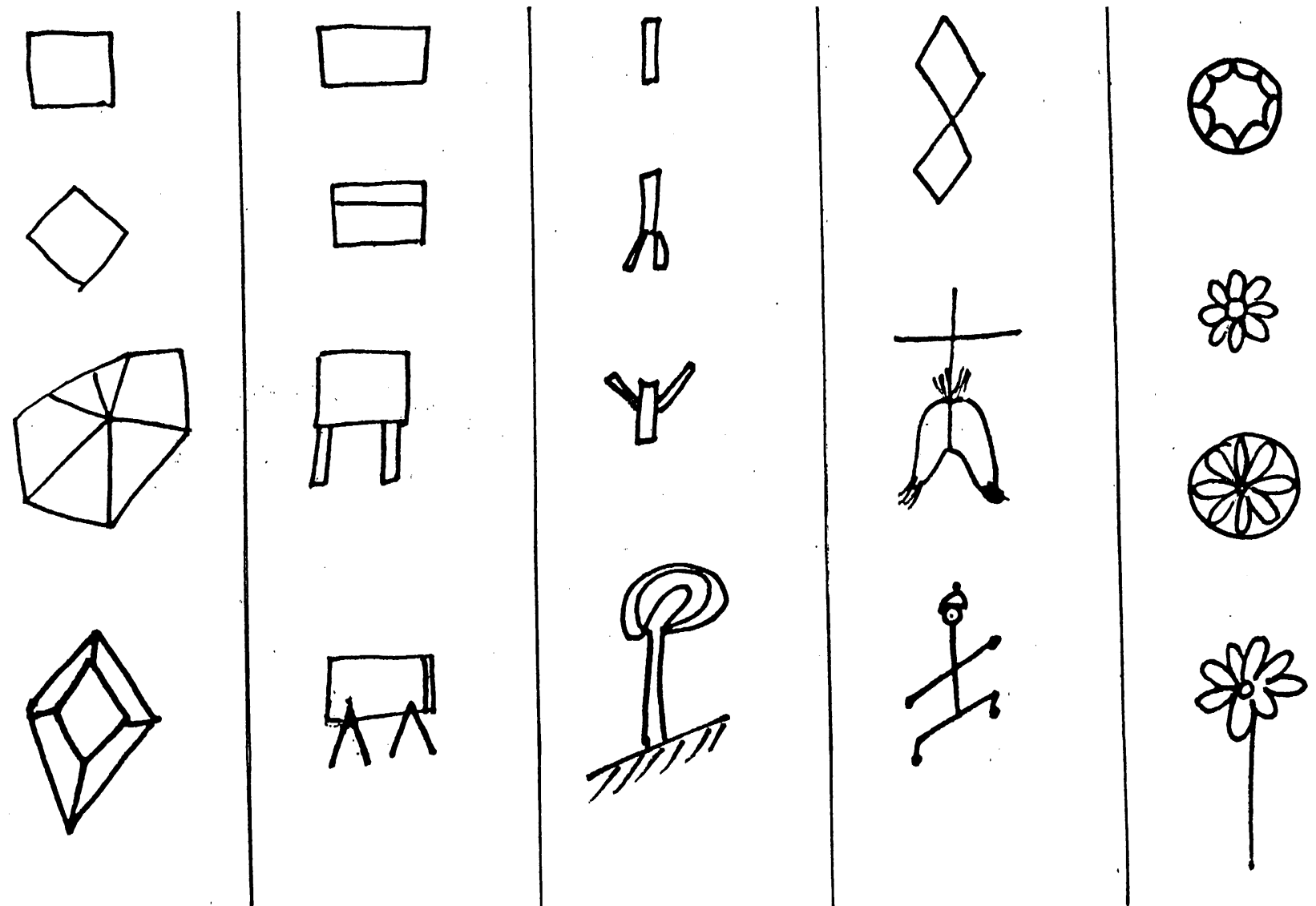

FIG. 4. Drawings of a cube, a table, a tree, a man and a flower by patients after right UES. Activity of the left hemisphere was retained.

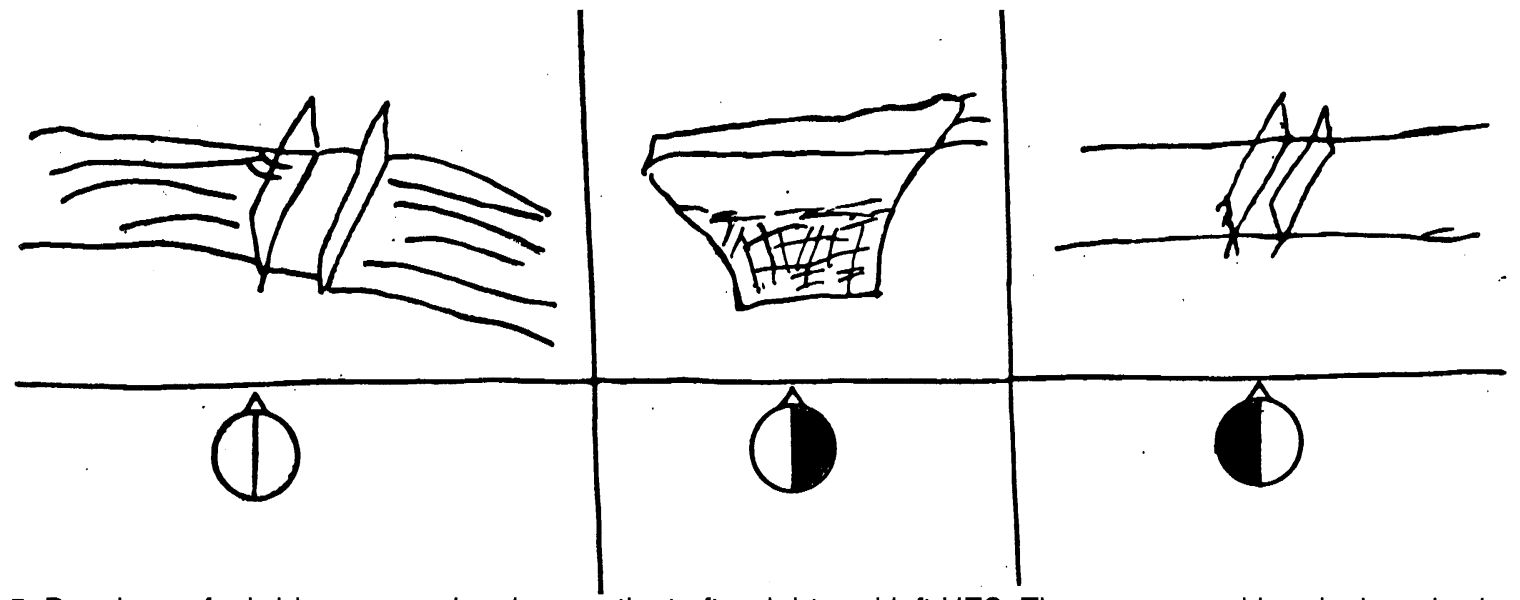

FIG. 5. Drawings of a bridge over a river by a patient after right and left UES. The suppressed hemisphere is shaded.

cally stylised representations of a person or a tree as as a cross were produced (Figure 4). In such geometrical signs-symbols, a system of binary space section was traced which reflected the oppositions of 'top-bottom' and 'left-right'. Such space section was also evident in representations of a flower when seen from above. The flower centre was usually surrounded by four or eight petals (Figure 6). The planar solution showed a conventional reflection of objective space geometry.

Under conditions of right hemisphere suppression, objects which are usually surrounded by people in near space, such as a table, were represented as if they were seen in the distance, in a plane, bodiless and generalised. The exaggerated direct perspective appeared 


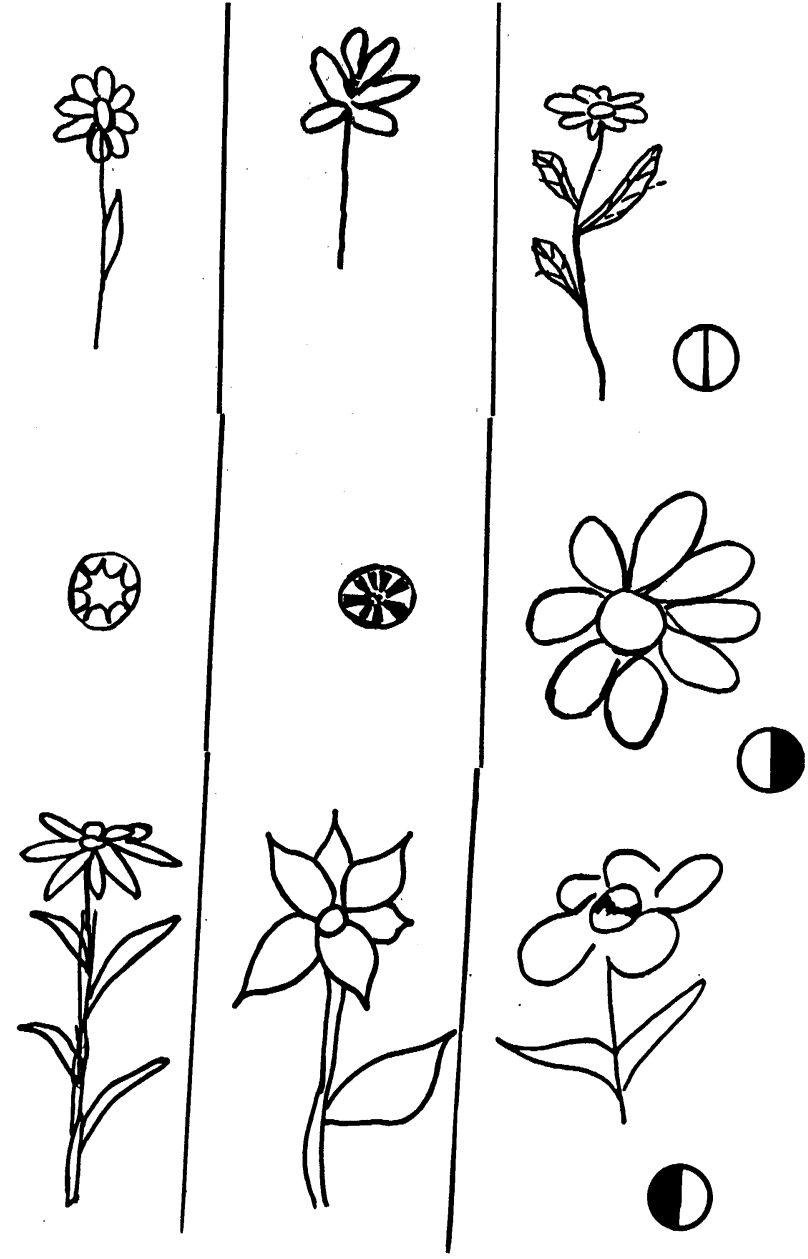

FIG 6. Drawings of a flower by three patients. The suppressed hemisphere is shaded.

fairly frequently, being present in $75 \pm 4 \%$ of drawings produced under right hemisphere suppression in comparison with $55 \pm 6 \%$ in control patients; $p<0.01$. To reproduce distant space in drawings, perspective was shortened and the size of objects in the distance was increased. For example, rails receding into the distance were transposed to an area within the zone of clear vision, i.e. in the near space. In such constructions, there is a convention that rails should converge at the horizon, i.e. in the far space. Often a 'bird's-eye' view or a distant view point was used; the rails were designated conventionally by lines only with no attempts to reproduce their visible shape when at close quarters (Figure 2). Under conditions of right hemisphere suppression, an attempt was made to reproduce direct perspective in drawings of a house at close quarters and a house in the distance. According to a verbal report by the patient, the lines connecting the houses 'are necessary in order to reduce the size of the house that is farther away' (Figure 7). When pooled data are considered, the house at close quarters was much reduced in size and it was moved upwards and to the right, towards the house in the distance (Figure 3). When the differences in size and distance are ignored, there is a shift of both representations to the distant part of space.

Separation and shifts in the parts of the representation in relation to each other were typical for right hemisphere suppression. Proportions of different parts of the object represented were crudely distorted. In other words, destruction of those components of perception which form the so-called visible geometry of objects in the world was reflected in the drawings (Deglin et al., 1987).

\section{Effects of psychotropic drugs on drawings}

It should be mentioned that the majority of drawings performed by patients before drugs were administered were stereotypes, such as the representation of a chamomile when the task was to draw a flower, and the drawings were rather resistant to the influence of the drugs.

Mood improving drugs. Changes were observed frequently after diazepam injection but they were observed most infrequently after injection of imipramine (Table II). In these drawings, the objective geometry of objects in the world was represented reliably more often under the influence of drugs than in the control situation. A tendency to schematise was noted and sensual authenticity and vividness were lost.

TABLE II. Frequency (\%) of representations of visible and objective geometry of space after injection with psychotropic drugs

\begin{tabular}{|c|c|c|c|c|}
\hline Drug & $\begin{array}{l}\text { Number of } \\
\text { drawings }\end{array}$ & $\begin{array}{c}\text { Objective } \\
\text { geometry }(\mathrm{A})\end{array}$ & $\begin{array}{c}\text { Visible } \\
\text { geometry }(B)\end{array}$ & $\begin{array}{l}\text { Without } \\
\text { changes }\end{array}$ \\
\hline diazepam & 92 & $26 \pm 5 * \star *$ & $5 \pm 2$ & $69 \pm 6$ \\
\hline imipramine & 94 & $11 \pm 3^{\star *}$ & $2 \pm 1$ & $87 \pm 3$ \\
\hline sodium amytal & 94 & $17 \pm 4^{\star \star}$ & $5 \pm 2$ & $73 \pm 4$ \\
\hline chlorpromazine & 100 & $4 \pm 2^{\star \star \star}$ & $19 \pm 4$ & $77 \pm 4$ \\
\hline haloperidol & 82 & $2 \pm 2^{* *}$ & $18 \pm 4$ & $80 \pm 4$ \\
\hline
\end{tabular}

${ }^{* *} \mathrm{P}_{\mathrm{A}-\mathrm{B}}<0.01 ; * * * \mathrm{P}_{\mathrm{A}-\mathrm{B}}<0.001$. 


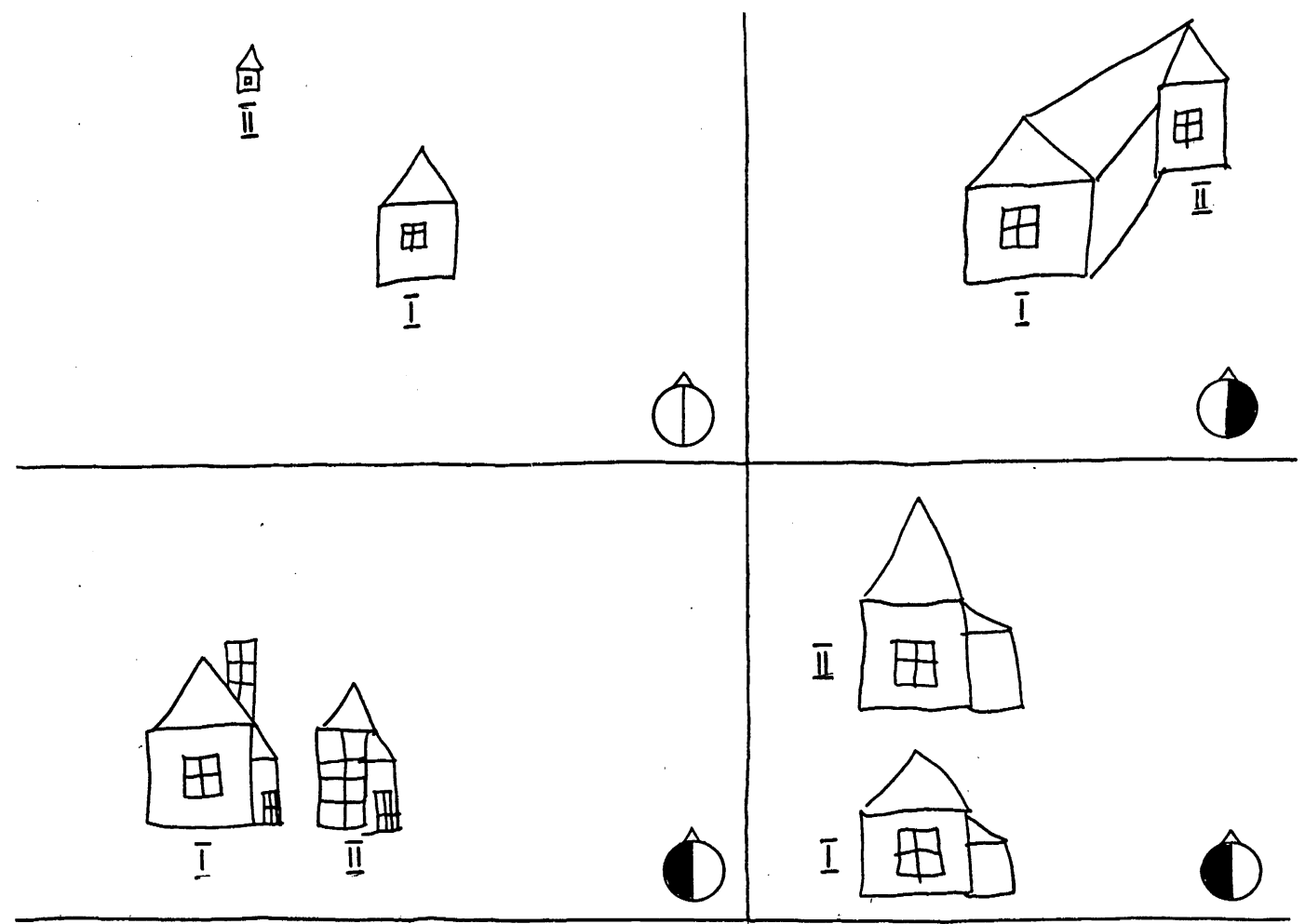

FIG. 7. Drawings of near (I) and distant (II) houses by a patient during the course of ECT. The suppressed hemisphere is shaded.

The methods of technical drawing were evident, as well as orthogonal projections, reamers and representation of invisible outlines. Decrease in the size of objects was also observed and pronounced shortening of perspective was evident.

Representations of a person after diazepam injection became more schematic and invisible parts of the body were drawn under the clothes as if they were seen by X-ray. The drawing itself was reduced in size and acquired a planar character. In the drawing of a house, a typical case of reamer was observed when the invisible side of the object was reproduced (Figure 8). Similar changes in the prevailing concepts underlying the drawings were observed after injection of imipramine and sodium amytal.

Neuroleptics. There was a tendency for changes in drawings to be observed more often following chlor- promazine injection than following haloperidol injection (Table II). The drawings reflected some features of objective geometry more frequently following drug administration than in the control situation (Table II). In drawings produced prior to neuroleptic injection, there were elements of sketchiness, as well as utilisation of the methods of technical drawing and shortening of perspective. After neuroleptic injection, representations acquired greater substance, volume and a greater resemblance to the object. In the drawings of a table, rails receding into the distance and a house at close quarters and a house in the distance, a reverse perspective as observed.

In the representation of a person, the extreme sketchiness was pronounced prior to drug administration. After chlorpromazine injection, the person acquired more pronounced features and the representation

TABLE III. Frequency (\%) of representation of visible and objective geometry of space in the drawings of depressed and manic patients

\begin{tabular}{lccc}
\hline Affective state & $\begin{array}{c}\text { Signs of objective } \\
\text { geometry }(\mathrm{A})\end{array}$ & $\begin{array}{c}\text { Signs of visible } \\
\text { geometry }(\mathrm{B})\end{array}$ & Without clear signs \\
\hline Depression & $28 \pm 3^{\star \star \star}$ & $57 \pm 3$ & $15 \pm 2$ \\
Mania & $62 \pm 4^{\star \star \star}$ & $20 \pm 3$ & $18 \pm 3$ \\
\hline
\end{tabular}

$\star \star \star P_{A-B}<0.001$

56 Behavioural Neurology • Vol 10 • 1997 


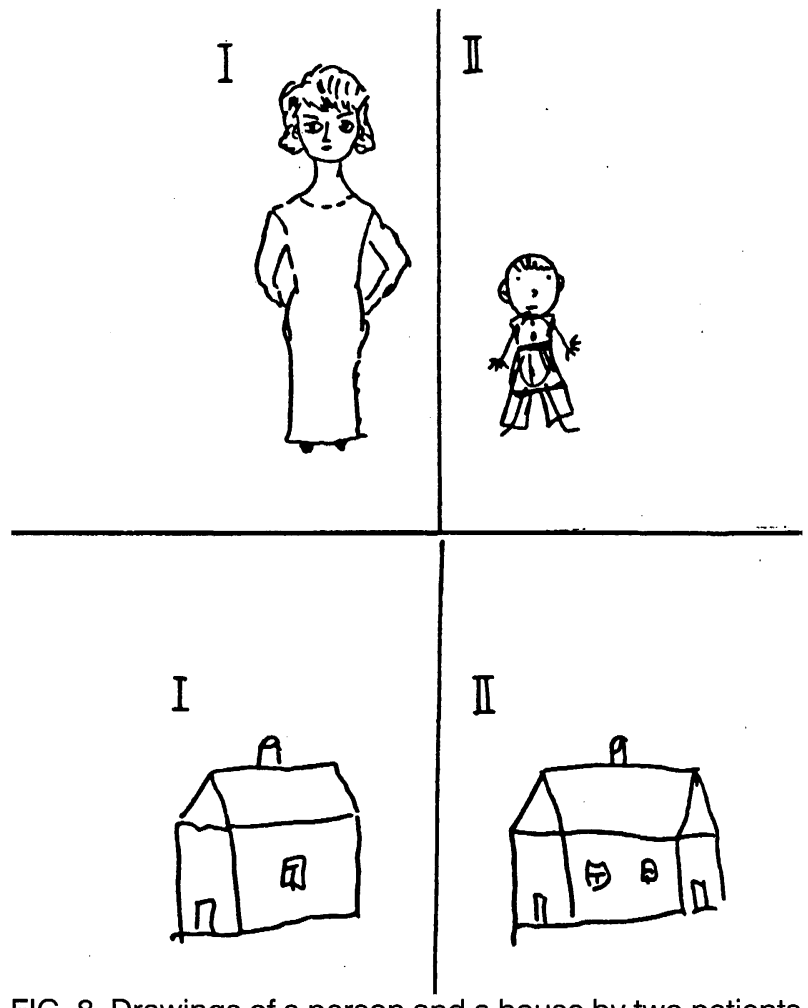

FIG. 8. Drawings of a person and a house by two patients (I) before and (II) after diazepam injections.

became more volumetric and vivid. In addition, the drawing was bigger. In the drawing of a house, the invisible side was represented before chlorpromazine injection. After the drug had been administered, the reamer of the house disappeared, although the schematic features remained (Figure 9). Changes towards prevailing evocative principles were also observed after haloperidol injection.

\section{Effects of affective disorders on drawings}

Depressed patients showed the same tendencies in the spatial constructions of drawings as those that were observed under the conditions of left hemisphere suppression and after neuroleptic injections. Indications of visible geometry were observed more frequently than indications of objective geometry (Table III). Spatial constructions performed by manic patients resembled the drawings performed under right hemisphere suppression and after injections of mood improving drugs. A prevalence of objective geometry signs was observed in this group (Table III).

Figure 10 shows drawings of objects by three patients who were tested in both depressed and manic states. In comparison with the depressed state, the drawings became more schematic with the signs of

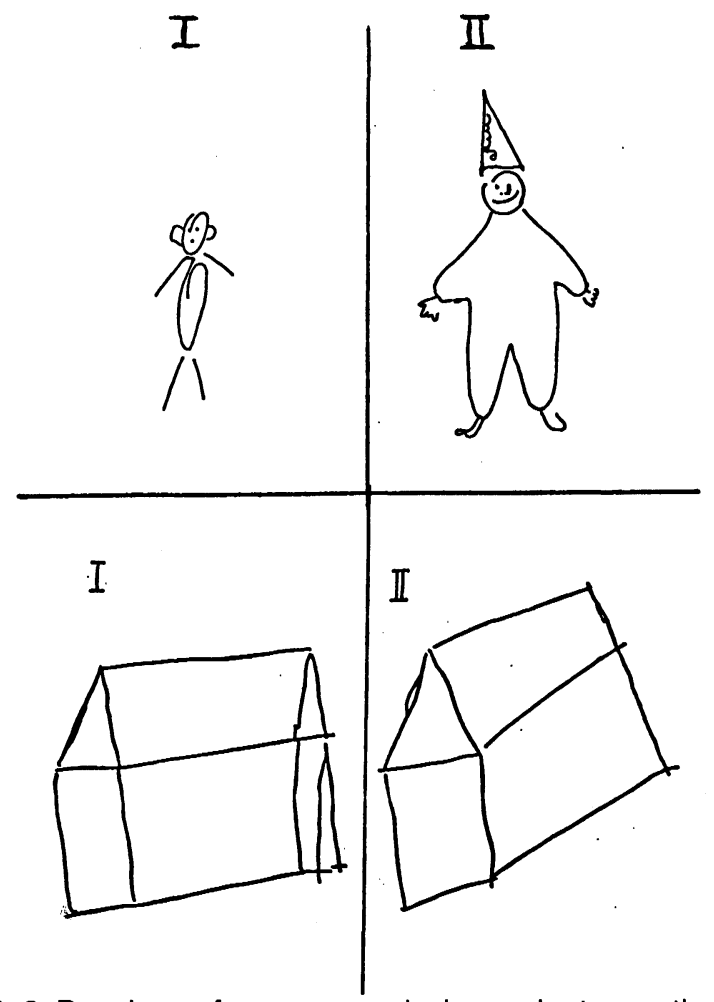

FIG. 9. Drawings of a person and a house by two patients (I) before and (II) after chlorpromazine injections.

exaggerated direct perspective. In the drawing of two houses, the shortening of perspective was evident in a dramatic decrease of the size of the distant house. Similar findings were apparent in drawings of a table and in drawings of rails receding into the distance. Shortening of perspective is characterised, in particular, by rails narrowing to a point in mania. When depressed, the patients preferred to create visual images of objects whereas in the manic state, they preferred to create schematic drawings with shortening of perspective.

\section{DISCUSSION}

Our results show that, under various conditions of brain activity, different and mutually complementary methods of space representation arise. In some states (left hemisphere suppression with right hemisphere activity; neuroleptic injection; depression), the creation of a visual image of a volumetric object from the point of view of routine vision is characteristic. Synthesis of different projections provides evidence of an ability to integrate the various sides of an object from different view points in the process of mentally rotating an object. The ability of the right hemisphere 
to provide the mental rotation in the process of representation of perception has been reported earlier (Ditunno and Mann, 1990).

Since the geometry of an object changes depending on the view point, representations of visible geometry created by the right hemisphere are also reflections of the perceptive space. In other words, the right hemisphere is responsible for the conception of perceptive space in man.

Lessening of the shortened perception of distant objects, moving the distant plane closer to the front one and employment of reverse perspective are also typical of right hemisphere activity. The reverse perspective is a particular case of the so-called perceptive perspective (Rauschenbach, 1980). It is restricted by the space near to the observer. With retained activity of the right hemisphere, continuity of size perception was observed only in the near space (Menshutkin and Nikolaenko, 1987). In addition, it was shown that the right hemisphere was responsible for the three-dimensional perception of an object in near space (Dobrovolskaya et al., 1995). It seems that the right hemisphere allows representation of volume and depth in space using several mechanisms, such as the vision of three-dimensional space and continuity of size and shape of objects. With the help of these mechanisms, the right hemisphere reconstitutes the visible geometry of a restricted (near) part of the space. Reflection of near space geometry by the right hemisphere secures regional orientation, which is responsible for immediate activity in man.

A destruction of those components of perception which form visible geometry of material objects is typical of the right hemisphere suppression, injection of drugs that improve the mood and mania. The mechanisms of continuity of size and shape of objects and of vision stereoscopy are altered and the three-dimensional world near the observer is represented as flat. Object representation looses sensual authenticity and realistic features in reflection of objects disappear. Thus, right hemisphere suppression results in a loss of the direct visualised character of visual perception. However, under these conditions, knowledge of objective properties of space is preserved. Representations of objective space geometry created by the left hemisphere were schematic. Only certain classes of objects were recognised under these conditions. The same abstract simplified forms, such as a rectangle, are used to designate different objects. A vertically-orientated rectangle represents a tree and a rectangle which is orientated horizontally represents a table. The image of a concrete object is substituted by its hieroglyph and by its generalised designation as the drawing idea. A

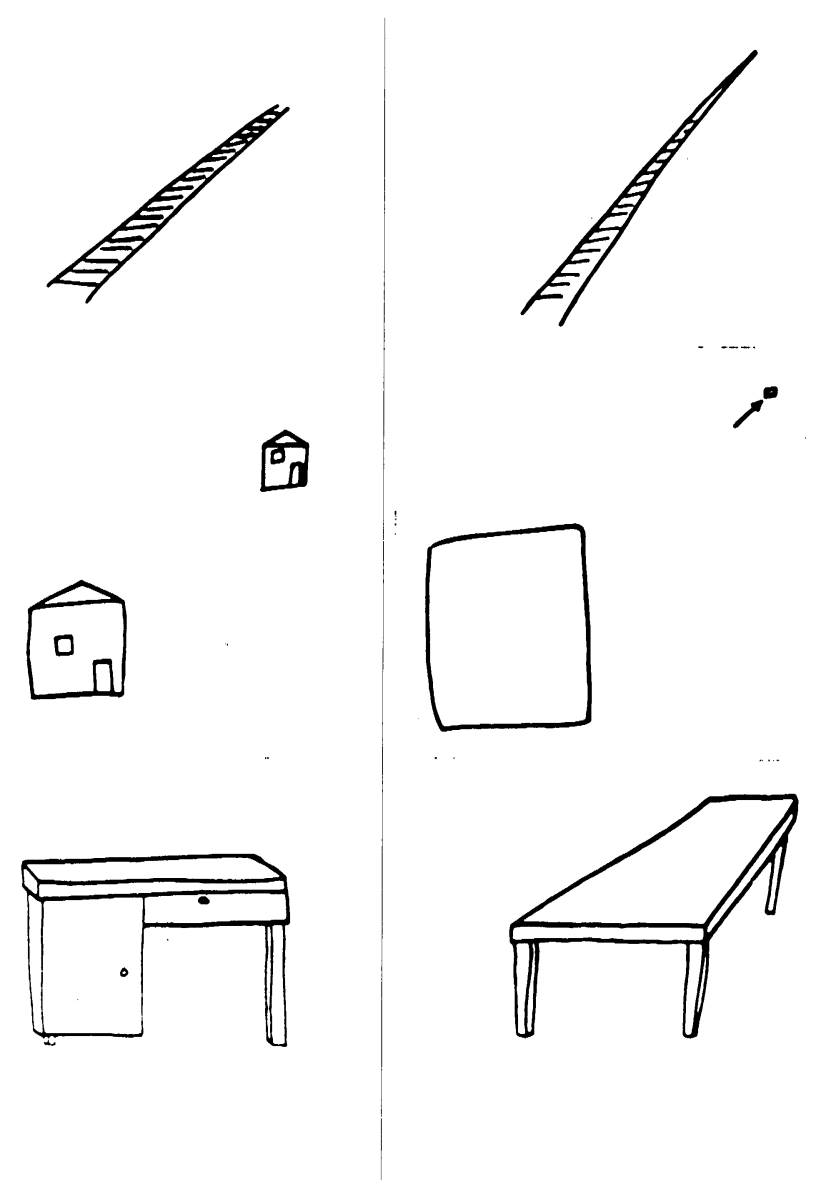

FIG. 10. Drawings of objects by three patients in depressed (left) and manic (right) states.

tendency to reflect knowledge of the objective geometry leads to employment of the methods of technical drawing including: orthogonal projections, reamers, representations of the invisible outline of the object and sections. It seems that, with the help of the left hemisphere, the subject represents a conception of the object rather than the object itself.

Finally, the left hemisphere utilises an excessively pronounced direct perspective. It seems that the left hemisphere is attempting to express conventionally the concept of the far space extension. When an object is perceived to be in real space, the left hemisphere shifts the object into far space (Dobrovolskaya et al., 1995).

It is possible that the left hemisphere is modelling objective space in an analytical way by means of sectioning it into discrete parts according to the principle of opponent definitions. If this is the case, there is a dichotomy in the operation of the left hemisphere which results in the representation of more complex geometrical figures, such as a cross, rectangle or rosettes, in a complex space. On the basis of representation of the objective geometry of space, the left 
hemisphere creates geometrical sign-symbols and, in a more general sense, a concept of the main directions of space. In other words, it performs a logical reconstruction of the objective space, reflecting a concept of the properties of the object in space.

In general, unlike the right hemisphere, the left hemisphere is orientated to the reflection of the distant part of the space, i.e. consideration of the space for a potential or probable activity which is important in the programming of activities.

The results of this study have indicated that two different types of drawings are closely connected with different space perception by the right and left hemispheres. Recent publications describe two different types of visual attention. Global, wide-angle attention is characteristic of the right hemisphere whereas focal, narrow angle attention is typical for the left hemisphere (Fink et al., 1996; Halligan and Marshall, 1994). It is probable that the drawing method of a patient depends on the visual attention type which predominates in particular conditions, such as unilateral seizure, depression, mania and administration of psychotropic drugs. In everyday life, knowledge of the objective space is considered by both the right and left hemispheres of the human brain.

\section{REFERENCES}

Arrigoni G and De Renzi E (1964) Constructional apraxia and hemispheric locus of lesions. Cortex, 1, 170-197.

Asadova MS (1985) Hemispheric interrelations in the firm emotional state changes. Human Physiology, 11, 443-447 (In Russian).

Balonov LY, Barcan DV, Deglin VL, Nikolaenko NN, Savranskaya RG, Trachenko OP and Chernigovskaya TV (1979) Unilateral Electroconvulsive Seizure. Nauka, Leningrad (In Russian).

Balonov LY, Deglin VL, Kaufmann DA and Nikolaenko NN (1984) Functional asymmetry of the animal brain. Neuroscience and Behavioral Physiology, 14, 427-437.

Davidson RJ (1991) Cerebral asymmetry and affective disorders: A developmental perspective. In: Internalizing and Externalizing Expressions of Dysfunction, Rochester Symposium on Developmental Psychopathology, Volume 2 (Eds D Cicchetti and SL Toth), pp. 123-154, Hillsdale, NJ.

Deglin VL, Ivashina GG and Nikolaenko NN (1986) Role of dominant and nondominant hemispheres in space representation. In: Neuropsychological Analyses of Functional Brain Asymmetry (Ed ED Chomskaya), pp. 58-70. Nauka, Moscow (In Russian).

Deglin VL, Lebedev BA, Nikolaenko NN and Isakov MP (1987) Unilateral Electroconvulsive Therapy Methodological Recommendations. Nauka, Leningrad (In Russian).
Ditunno PL and Mann VA (1990) Right hemisphere specialization for mental rotation in normal and brain damaged subjects. Cortex, 26, 177-188.

Dobrovolskaya NV, Levitina EA and Egorov AY (1995) Depth perception and functional brain asymmetry. Sensory Systems, 9, 68-74 (In Russian).

Egorov AY (1986) Visual after images as a pattern of functional hemispheric asymmetry. Human Physiology, 12, 664-668 (In Russian).

Egorov AY (1989) The influence of seduxen (diazepam) and aminazine (chlorpromazine) upon hemispheric interaction in man. Human Physiology, 15, 128-134 (In Russian).

Egorov AY and Nikolaenko NN (1992) Functional brain asymmetry and visuo-spatial perception in mania, depression and under psychotropic medication. Biological Psychiatry, 32, 399-410.

Fink GR, Halligan PW, Marshall JC, Frith CD, Frackowiak RSJ and Dolan RJ (1996) Where in the brain does visual attention select the forest and the trees? Nature, 382, 626-628.

Flor-Henry P (1983) Cerebral Basis of Psychopathology. John Wright PSG INS, Boston, Bristol, London.

Flor-Henry P, Koles ZJ, Howarth BG and Burton L (1979) Neurophysiology study in schizophrenia, mania and depression. In: Hemisphere Asymmetries of Function in Psychopathology, (Eds J Gruzelier and P. Flor-Henry) pp. 189-221. Elsevier/North Holland Biomedical Press, Amsterdam.

Halligan PW and Marshall JC (1994) Towards a principled explanation of unilateral neglect. Cognitive Neuropsychology, 11, 167-206.

Menshutkin VV and Nikolaenko NN (1987) The role of the right hemisphere in maintenance of constancy perception of object sizes. Human Physiology, 13, 324-326 (In Russian).

Nikolaenko NN and Deglin VL (1984) Space semotics and functional asymmetry of the brain. In: Dialog Structure as a Work Principle of Semiotic Mechanism. Transactions on Sign Systems Volume 17. Scientific Papers of Tartu State University, N. 641.pp. 48-67, Tartu (In Russian).

Piercy M, Hecaen $H$ and Ajuriaguerra J (1960) Constructional apraxia associated with unilateral cerebral lesions. Brain, 83, 225-242.

Rauschenbach BV (1980) Space constructions in painting. Nauka, Moscow (In Russian).

Schweitzer L (1979) Differences of cerebral lateralization among schizophrenic and depressed patients. Biological Psychiatry, 14, 721-733.

Warrington EK, James M and Kinsbourne M (1966) Drawing disability in relation to laterality of cerebral lesions. Brain, 89, 53-82.

Wexler BE and Heninger GR (1979) Alterations in cerebral laterality during acute psychotic illness. Archives of General Psychiatry, 36, 278-284.

(Received 11 April 1996; accepted as revised 27 May 1997) 


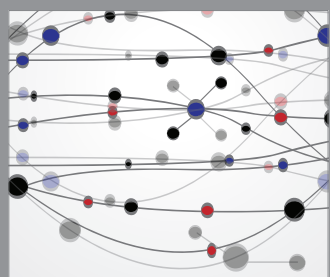

The Scientific World Journal
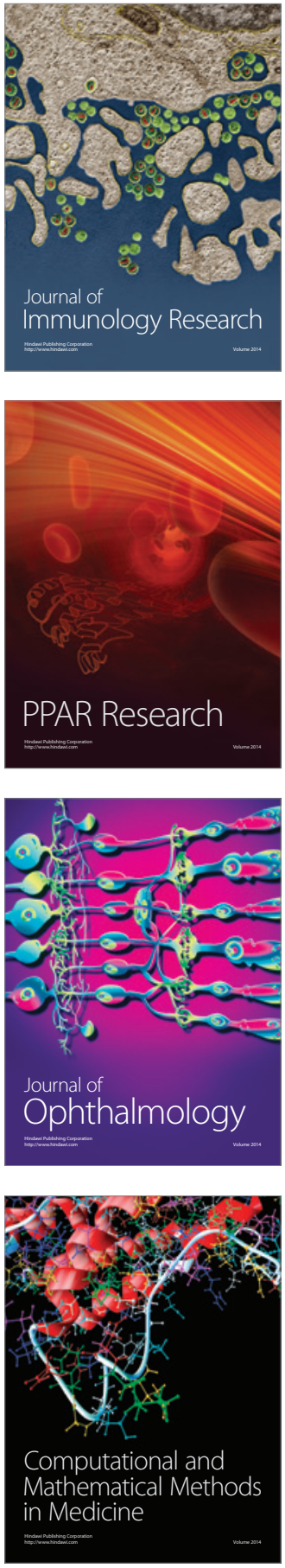

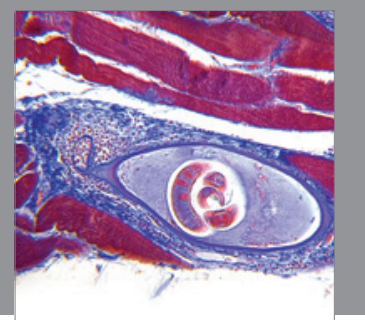

Gastroenterology

Research and Practice
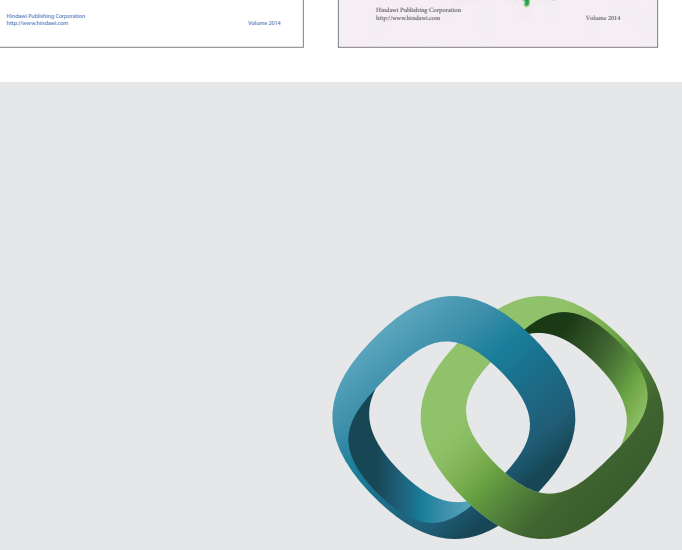

\section{Hindawi}

Submit your manuscripts at

http://www.hindawi.com
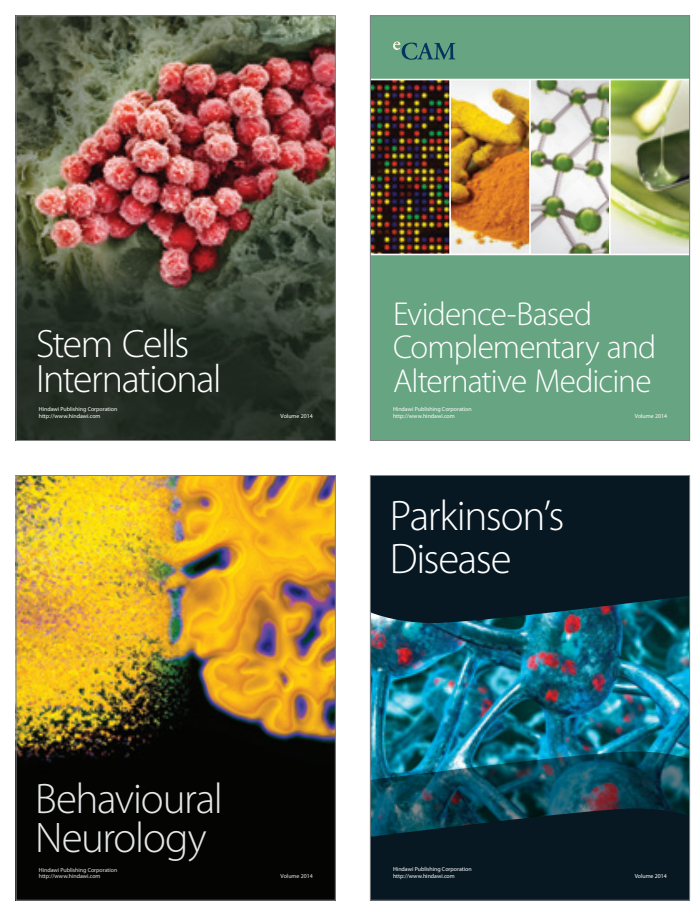

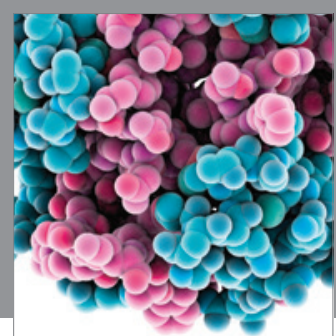

Journal of
Diabetes Research

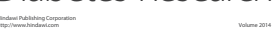

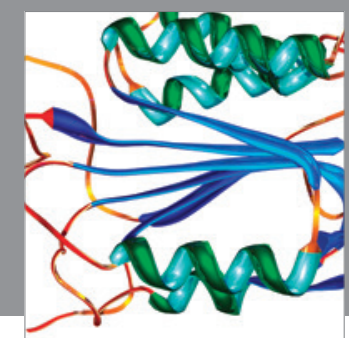

Disease Markers
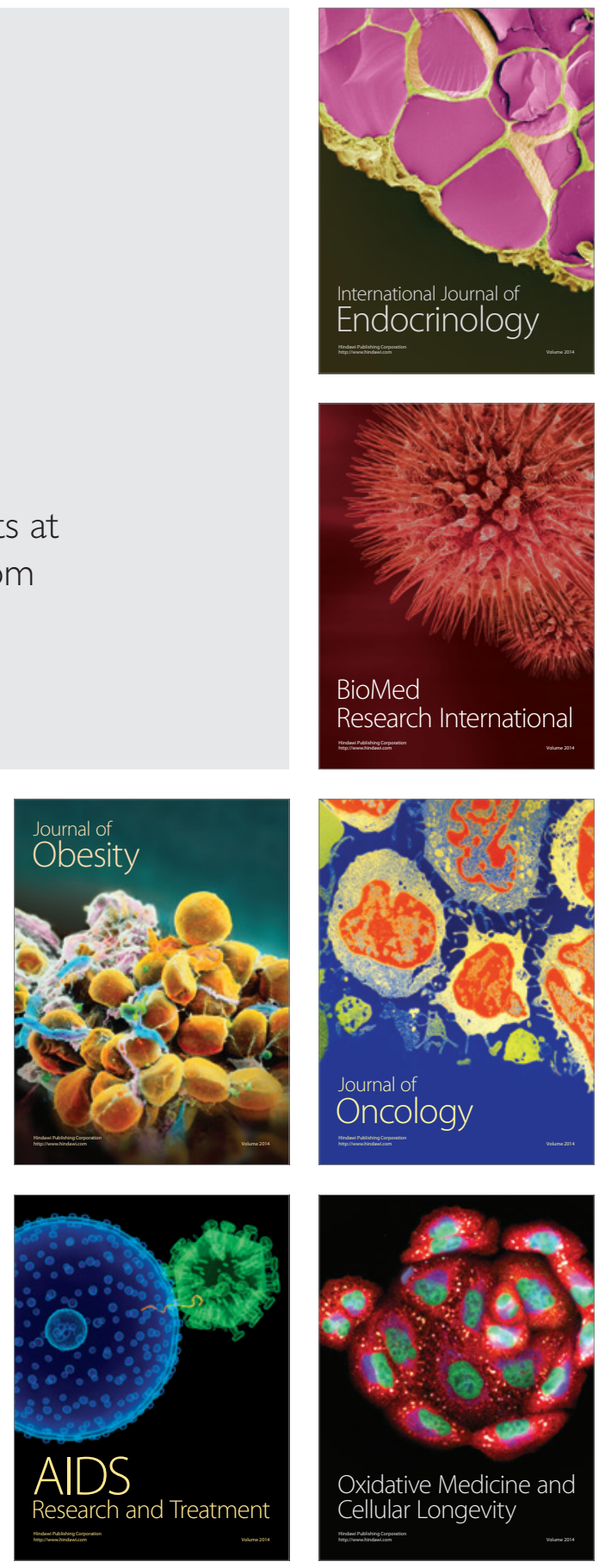